\section{Characterization of AcMNPV with a deletion of ac69 gene}

\author{
Jianhao Ke, ${ }^{1,2}$ Jinwen Wang, \\ Riqiang Deng, ${ }^{1}$ Lin Lin, 1,3 Bei Jinlong,,2 \\ Liao Yaoping, ${ }^{2}$ Xunzhang Wang ${ }^{1}$ \\ 1State Key Laboratory of Biocontrol, \\ School of Life Sciences, Sun Yat-sen \\ University, Guangzhou; ${ }^{2}$ Rice Research \\ Institute, Guangdong Academy of \\ Agricultural Sciences, Guangzhou; \\ ${ }^{3}$ Shenzhen Academy of Metrology and \\ Quality Inspection, Shenzhen, China
}

\section{Abstract}

ORF69 (Ac69) of Autographa californica multiple nucleopolyhedrovirus (AcMNPV) is conserved in some baculovirus genomes. Although it has been shown that Ac69 has cap 0 -dependent methyltransferase activity and is not required for budded virus production in Spodoptera frugiperda Sf-9 cells, its role in occlusion-derived virus synthesis and virus oral infectivity is not known. This paper describes generation of an ac69 knockout AcMNPV bacmid mutant and analyses of the influence of $a c 69$ deletion on the viral infectivity in Sf-9 cells and Trichoplusia $n i$ larvae so as to investigate the role of $a c 69$ in the viral life cycle. Results indicated that ac69 deletion has little effect on the production rates and morphogenesis of budded virus and occlusionderived virus in Sf-9 cells. In addition, animal experiment revealed that the deletion mutant did not affect AcMNPV infectivity for Trichoplusia ni larvae in $\mathrm{LD}_{50}$ and $\mathrm{LT}_{50}$ bioassay when administered orally. These results suggest that ac69 may be dispensable for viral infectivity both in vitro and in vivo.

\section{Introduction}

Autographa californica multiple nucleopolyhedrovirus (AcMNPV) is the most intensively studied member of the Baculovirade family, which encompasses a group of arthropod-specific DNA viruses with a circular covalently closed genome of 80 to $180 \mathrm{~kb}^{1-3}$ During baculovirus infection cycle, gene transcription is characteristic of early, late, and very late phases. By the host RNA polymerase II, the early gene transcription is initiated from a highly conserved TATA motif which is found in most early gene promoters or from other cis-acting regulatory elements, such as the initiator element CAGT motif. ${ }^{4-6}$ By the virus-encoded RNA polymerase, the late and very late gene tran- scription is initiated from a highly conserved motif containing the sequence (A/G/T) TAAG that is also found in most late and very late gene promoters. ${ }^{7}$

Two viral forms, budded virus (BV) and occlusion-derived virus (ODV), are produced during the viral infection cycle. ${ }^{8}$ ODV is responsible for horizontal transmission between insect hosts, whereas BV is responsible for systemic spread through the insect host and propagation in tissue culture. ${ }^{8}$ Based on the nature of the viral genome, the genes encoded by baculovirus can be divided into two broad groups, essential genes and auxiliary genes. ${ }^{9}$ The former is essential for viral replication, while the latter is not required for viral propagation and just provide virus with some selective advantage in nature.

orf69 (ac69) gene of AcMNPV encodes a protein of amino acids with a molecular mass of $30.355 \mathrm{kDa}$. Among the forty-four baculoviruses that have been sequenced to date, thirty-seven nucleopolyhedroviruses (NPVs) but no granulovirus (GVs) have an Ac69 homolog. The Ac69 protein is highly conserved in AcMNPV, Rachiplusia ou NPV, Bombyx mori NPV and Maruca vitrata NPV. The amino acid identities of Ac69 among these four NPVs are $87-100 \%$, while the amino acid identities between Ac69 and its homologs of other NPVs are $33-62 \%$.

InterProScan program shows that Ac69 homologs constitute a protein family called FtsJ-like methyltransferase family (IPR0096 15). This family consists of FtsJ from various bacterial and archaeal sources. FtsJ is a methyltransferase, but actually has no effect on cell division. FtsJ's substrate is the $23 \mathrm{~S}$ rRNA. The $1.5 \mathrm{~A}$ crystal structure of FtsJ in complex with its cofactor S-adenosylmethionine revealed that FtsJ has a methyltransferase fold. This family also includes the $\mathrm{N}$ terminus of flaviviral NS5 protein. It has been hypothesised that the N-terminal domain of NS5 is a methyltransferase involved in viral RNA capping.

Previous report has shown that ac69 can stimulate late gene expression in a transientexpression assay system. ${ }^{10} \mathrm{Wu}$ and coworkers showed that Ac69 which was synthesized during the late phase of infection had cap 0 dependent methyltransferase activity. ${ }^{11}$ Characterization of a virus lacking ac69 indicated that it was infectious when infected into Sf-9 cells and single-step viral growth curves revealed that ac69 deletion did not affect budded virus production in tissue culture. However, the function of Ac69 in ODV formation and virus oral infectivity has not been determined.

Therefore, to continue the functional investigation of Ac69, we generated an ac69 knockout AcMNPV virus to examine the role of this gene using the AcMNPV bacmid system. The results described herein demonstrate that ac69 deletion mutant had no striking pheno-
Correspondence: Xunzhang Wang, State Key Laboratory of Biocontrol, School of Life Sciences, Sun Yat-sen University, 510275 Guangzhou, China.

Fax +86.20.84113964 - Tel: +86.20 .84113964$

E-mail:wxz@mail.sysu.edu.cn

Key words: Baculovirus, orf69, AcMNPV, Auxiliary gene.

Acknowledgments: we thank Dr. Yi Luo for providing useful suggestion during the course of the study. This work was funded by the Natural Science Foundation of Guangdong Province under Grant 4203388 and President Foundation of Guangdong Academy of Agricultural Sciences under Grant 20090102.

Received for publication: 20 June 2011.

Revision received: 2 July 2011.

Accepted for publication: 7 July 2011.

This work is licensed under a Creative Commons Attribution NonCommercial 3.0 License (CC BYNC 3.0).

(C) Copyright J. Ke et al., 2011

Licensee PAGEPress, Italy

Microbiology Research 2011; 3:e4

doi:10.4081/mr.2011.e4

type in Spodoptera frugiperda Sf-9 cells, including BV and ODV production, cytological changes and viral morphogenesis, but also in Trichoplusia $n i$ (T. ni) larvae, including 50\% lethal dose $\left(\mathrm{LD}_{50}\right)$ and $50 \%$ lethal time $\left(\mathrm{LT}_{50}\right)$ bioassays.

\section{Materials and Methods}

\section{Cells, viruses and primers}

The insect Spodoptera frugiperda cells line Sf9 cells were cultured at $27^{\circ} \mathrm{C}$ in Grace's medium (Invitrogen Life Technologies) supplemented with $10 \%$ fetal bovine serum, penicillin (100 $\mu \mathrm{g} / \mathrm{mL})$ and streptomycin $(30 \mu \mathrm{g} / \mathrm{mL})$. Bacmid bMON14272 (Invitrogen Life Technologies), containing the AcMNPV genome, can propagate in Escherichia coli strain DH10B as described previously. ${ }^{12}$ All sequences of the primers used in this study are shown in Table 1.

\section{Construction of ac69 knockout AcMNPV bacmid}

Strategy for construction of an ac69-null bacmid lacking intact ac69 gene is shown in Figure 1A. With primers ac69U1 and ac69U2, a 579-bp fragment (at AcMNPV genome position 58841-59399) homologous to the upstream sequence of ac69 was PCR amplified from AcMNPV genome. The resulting product was 
digested with HindIII/NotI and then cloned into HindIII/NotI digested vector pET28a- $\mathrm{Z}^{13}$ to generate the recombinant plasmid pET28a-USZ. With primers $a c 69 \mathrm{D} 1$ and $a c 69 \mathrm{D} 2$, a 727-bp fragment (at AcMNPV genome position 5990260609) homologous to the downstream sequence of ac69 was PCR amplified from AcMNPV genome. The resulting product was digested with $\mathrm{Bam} \mathrm{HI} / \mathrm{XbaI}$ and then cloned into Bam $\mathrm{HI} / X$ baI digested vector pET28a-US-Z to generate the recombinant plasmid pET28a-USZ-DS. The pET28a-US-Z-DS was digested with NotI/XbaI. The resulting linear 2098-bp fragment containing Zeocin and $a c 69$ flank regions was gel purified and suspended in distilled water. Next, an AcMNPV ac69 knockout bacmid was generated by using a modification of the $\lambda$ phage Red recombinase system as described previously. ${ }^{13}$ The resulting AcMNPV ac69 knockout bacmid was named Ac-ac69-KO.

The deletion of ac69 from the AcMNPV bacmid genome was confirmed by PCR with two primer pairs. Primers REP1 and REP2 were used to confirm the partial deletion and the insert of Zeocin gene within ac69 coding region, while Primers ComfirmP1 and ZeoP2 were used to confirm the correct junction between Zeocin and upstream. The relative positions of these two primer pairs in Ac-ac69$\mathrm{KO}$ bacmid were illustrated in Figure 1B.

\section{Construction of knockout, repair and wt AcMNPV bacmids with \\ polyhedrin and green fluorescent protein}

As described previously, ${ }^{13}$ two marker genes, polyhedrin and $g f p$, were introduced into the polyhedrin locus of ac6 9 knockout, repair and wt bacmids by $\mathrm{Tn} 7$ mediated transposition (Figure 1C). With Primers REP1and REP2, a 1454-bp Xbal/XhoI fragment (at AcMNPV genome position 59112-60547) containing the wt $a c 69$ gene with its own promoter and poly (A) tail was PCR amplified. The resulting prod-

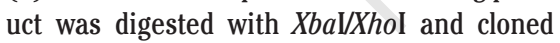
into $X b a \mathrm{I} / X h o I$ digested $\mathrm{pFB}$-ieGP ${ }^{14}$ to generate pFB-ieGP-ac69. The electro-competent DH10B cells harboring wt bacmid and the helper plasmid pMON7124 were transformed with pFBieGP to generate the wt control bacmid (AcGP). The electro-competent DH10B cells harboring Ac-ac69-K0 bacmid and the helper plasmid pMON7124 were transformed with pFBieGP and pFB-ieGP- $a c 69$ to generate the $a c 69$ knockout bacmid (Ac-ac69-K0-GP) and the ac69 repair bacmid (Ac-ac69-REP-GP), respectively. Strategy for confirmation of the all constructs by PCR analysis is shown in Figure 1D. Primers ComfirmP1 and ZeoP2 produced a single 1898-bp band in Ac-ac69-K0-GP and Acac69-REP-GP but no product in Ac-GP. Primers REP1 and REP2 produced single fragments of 1454 bp and 1746 bp for Ac-GP and Ac-ac69-KO-
GP, respectively, but both fragments for Acac69-REP-GP. Transposition event of polyhedrin and gfp marker genes was confirmed later by GFP expression and occlusion body formation in virus-infected Sf- 9 cells as described below. The correct recombinant bacmid was isolated and electroporated back into DH10B cell, and colonies were screened for sensitivity to tetracycline to ensure that the isolated bacmid was free of the helper plasmid.

\section{Virus growth curve}

For analysis of virus growth curve, Sf-9 cells ( $2 \times 10^{6} / 35$-mm-diameter dish) were transfected in triplicate with $2.0 \mu \mathrm{g}$ of each bacmid DNA (Ac-GP, Ac- $a c 69-\mathrm{KO}-\mathrm{GP}$ and Ac- $a c 69$-REP-GP)

Table 1. Primers used in this study.

\begin{tabular}{llc}
\hline Primers & \multicolumn{1}{c}{ Sequences $^{2}$} & Restriction enraymes \\
ZeoP1 & 5'GACAAGCTTAATCTAAGGGGCGGTGT & HindIII \\
ZeoP2 & 5'CATGGATCCTGCTGCTCACATGTTGGTCT & Bam HI \\
\hline ac69U1 & 5'TATGCGGCCGCCCGCTACTTTCTCCAGACAAA & NotI \\
ac69U2 & G 5'GTCAAGCTTAACGCCAACATCTAGGTCAG & HindIII \\
\hline ac69D1 & 5'CATGGATCCTTCTTCTCG GCCTGCCAATTC' & Bam HI \\
ac69D2 & 5'GACTCTAGAGGTTGATTGCAAACTTGGCACG & XbaI \\
\hline REP1 & 5'ACGTCTAGACAACGAGAGAATAAGAGCGG & XbaI \\
REP2 & 3'5'TTACTCGAGCGAAGGACAATGACCATCAATCG & Xhol \\
\hline ComfirmP1 & 5'CACCTCATTGATGTCGTCAGA & - \\
\hline
\end{tabular}

aThe restriction sites are underlined.

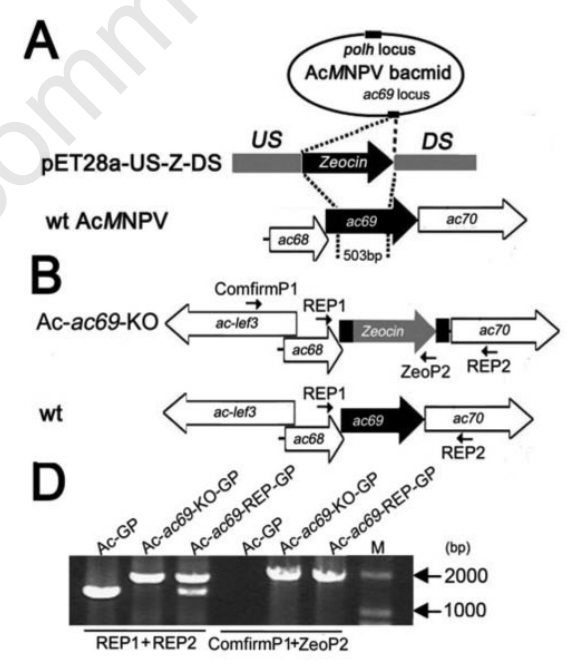

Figure 1. Construction of ac69 knockout, repair, and wt AcMNPV bacmids containing gfp and polyhedrin. (A) Schematic diagram for construction of an ac 69 knockout bacmid containing a deletion of the ac69 gene by recombination in E.coli. A 503-bp fragment of the ac69 orf was deleted and replaced by the Zeocin resistance gene under the control of the EM7 promoter. (B) Diagram indicating the positions of primer pairs used in the confirmation of disruption of ac69 and the correct insertion of Zeocin resistant gene in ac69 locus. (C) Schematic diagram of three viruses, Ac-GP, Ac-ac69-KO-GP and Ac-ac69-REPGP, showing the polyhedrin and gfp genes inserted into the polyhedrin locus by Tn7mediated transposition. (D) Confirmation by PCR analysis of the presence or absence of sequence modifications among each bacmid (Ac-GP, Ac-ac69-KO-GP and Ac-ac69-REPGP). The virus templates are shown above each lane, and the primer pairs used are shown below.

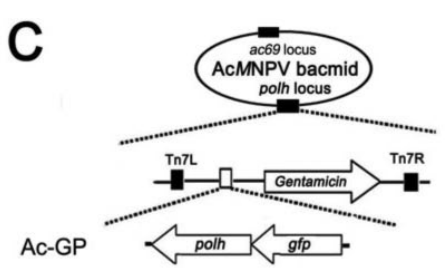

using Cellfectin liposome reagent (Invitrogen Life Technologies). Virus supernatant was coltiters were determined by $\mathrm{TCID}_{50}$ end point dilution assay in Sf-9 cells.15 Sf-9 cells were subsequently infected in triplicate with each
virus at an MOI of 5 and at designated time points the titers were determined by a $\mathrm{TCID}_{50}$

Electron microscopy analysis

$2 \times 10^{6}$ Sf-9 cells were infected with each 5. At 24 and 72 host-infection (p.i.), were dislodged and pelleted at $1000 \mathrm{~g}$ for 5 min. Cells were fixed, dehydrated, embedded,

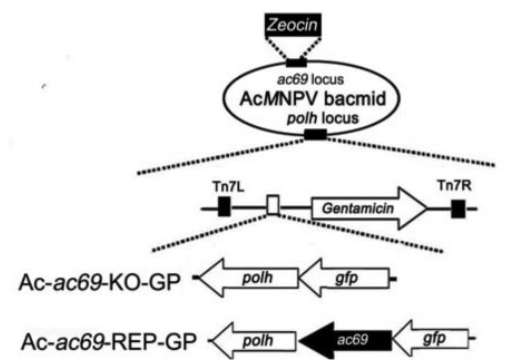
end-point dilution assay in Sf-9 cells. 
sectioned, and stained as described previously. ${ }^{14}$ Samples were observed under a JEM100CXII transmission electron microscope operation at 80 kilovolts.

\section{Bioassays}

Bioassays were performed to evaluate whether ac6 9 has any effect on the infectivity of AcMNPV. $2 \times 10^{6} \mathrm{Sf}-9$ cells were infected in triplicate with each virus (Ac-GP and Ac-ac69K0-GP) at an MOI of 5. Polyhedrin inclusion bodies (PIBs) were harvested at $120 \mathrm{~h}$ p.i. and then purified by centrifugation and resuspended in double-distilled water as described previously. ${ }^{15}$ Newly molted third-instar $T$. ni larvae were starved for $6 \mathrm{~h}$ and then were individually reared in 12-well plates with artificial diet containing each dosage $(0,25,50,100$ and 200 PIBs per larvae, respectively) for the $50 \%$ lethal does $\left(L_{50}\right)$ analysis or containing each dosage (0 and 200 PIBs per larvae, respectively) for the $50 \%$ lethal time $\left(\mathrm{LT}_{50}\right)$ analysis. Only the larvae that completely ingested the diet with virus within $12 \mathrm{~h}$ were further reared. Thirtysix larvae per dosage were used in the infection experiments and the experiments were repeated for three times. For $\mathrm{LT}_{50}$ analysis, mortality was recorded every $12 \mathrm{~h}$ after infection until larvae died or pupated. The $\mathrm{LD}_{50}$ and $\mathrm{LT}_{50}$ values were determined using the probit analysis.

\section{Results}

\section{Analysis of knockout, rescue, and wt AcMNPV bacmids replication in $\mathrm{Sf}-9$ cells}

To examine the effect of $a c 69$ deletion on viral propagation and occlusion synthesis, $\mathrm{Sf}-9$ cells were infected with each virus (Ac-GP, Acac69-KO-GP and Ac-ac69-REP-GP) at an MOI of 5 . Fluorescence due to the expression of $g f p$ in infected cells was monitored (Figure 2A). No difference among the three viruses was observed by $12 \mathrm{~h}$ p.i. Fluorescence was observed in almost all Ac-GP, Ac-ac69-KO-GP or Ac-ac69-REP-GP-infected cells by $48 \mathrm{~h}$ p.i., indicating all these viruses were able to produce infectious budded virus.

Microscopic analysis revealed that occlusion bodies were observed in approximately $40 \%$ of Ac-GP, Ac- $a c 69$-KO-GP or Ac- $a c 69$-REP-GPinfected cells by $36 \mathrm{~h}$ p.i., and appeared in nearly all of Ac-GP, Ac-ac69-K0-GP and Acac69-REP-GP-infected cells by $72 \mathrm{~h}$ p.i. (Figure $2 \mathrm{~B})$, implying that $a c 69$ deletion did not affect occlusion body synthesis.

To access the effect of $a c 69$ knockout on BV replication, a virus growth curve analysis was performed. The results revealed that Sf-9 cells infected with Ac-GP, Ac-ac69-K0-GP or Acac69-REP-GP showed steady increase in virus production and the slope of the growth curves of the three viruses had no significant difference (Fig. 2C), indicating ac 69 deletion had little effect on the efficiency of BV synthesis in Sf-9 cells.

\section{Electron microscopy analysis of ac69wt, knockout and mock-infect- ed Sf-9 cells}

To further analyze whether ac69 deletion has any effect on virus morphogenesis, Sf-9 cells infected with Ac-GP or Ac-ac69-KO-GP were examined by electron microscope. Results revealed that the nuclei of cells infected with either Ac-GP or Ac-ac69-KO-GP exhibited the typical baculovirus infection symptoms, i.e., enlarged nuclei, a typically reorganized electron-dense virogenic stroma (the putative site of nucleocapsids assembly) enriched with nucleocapsids, bundles of nucleocapsids aligning with envelopes and their subsequent envelopment to form ODV, and occlusion bodies containing ODVs appearing within the ring zone which is a significant and morphologically distinct peristromal compartment of nucleoplasm and yields along with maturation of the virogenic stroma, but no such structure in mock-infected cells. In addition, no distinguishing differences of nucleocapsids and occlusion bodies were observed between Ac-GP and Ac-ac69-KO-GP-infected cells. Electron microscopic analysis of Ac-ac69K0-GP-infected Sf-9 cells is shown in Figure 3. These observations indicated that ac69 deletion did not affect morphogenesis of BVs and ODVs in Sf-9 cells.

\section{Effect of ac69 deletion of AcMNPV on infectivity for Trichoplusia ni \\ larvae}

The infectivities of Ac-GP and Ac-ac69-KOGP were determined for newly molted threeinstar T.ni larvae in $50 \%$ lethal dose $\left(\mathrm{LD}_{50}\right)$ and $50 \%$ lethal time $\left(\mathrm{LT}_{50}\right)$ bioassays when administered orally (Tables 2 and 3). No significant difference in $\mathrm{LD}_{50}$ and $\mathrm{LT}_{50}$ was observed between the two viruses, suggesting ac6 6 is not required for viral infectivity in T.ni larvae
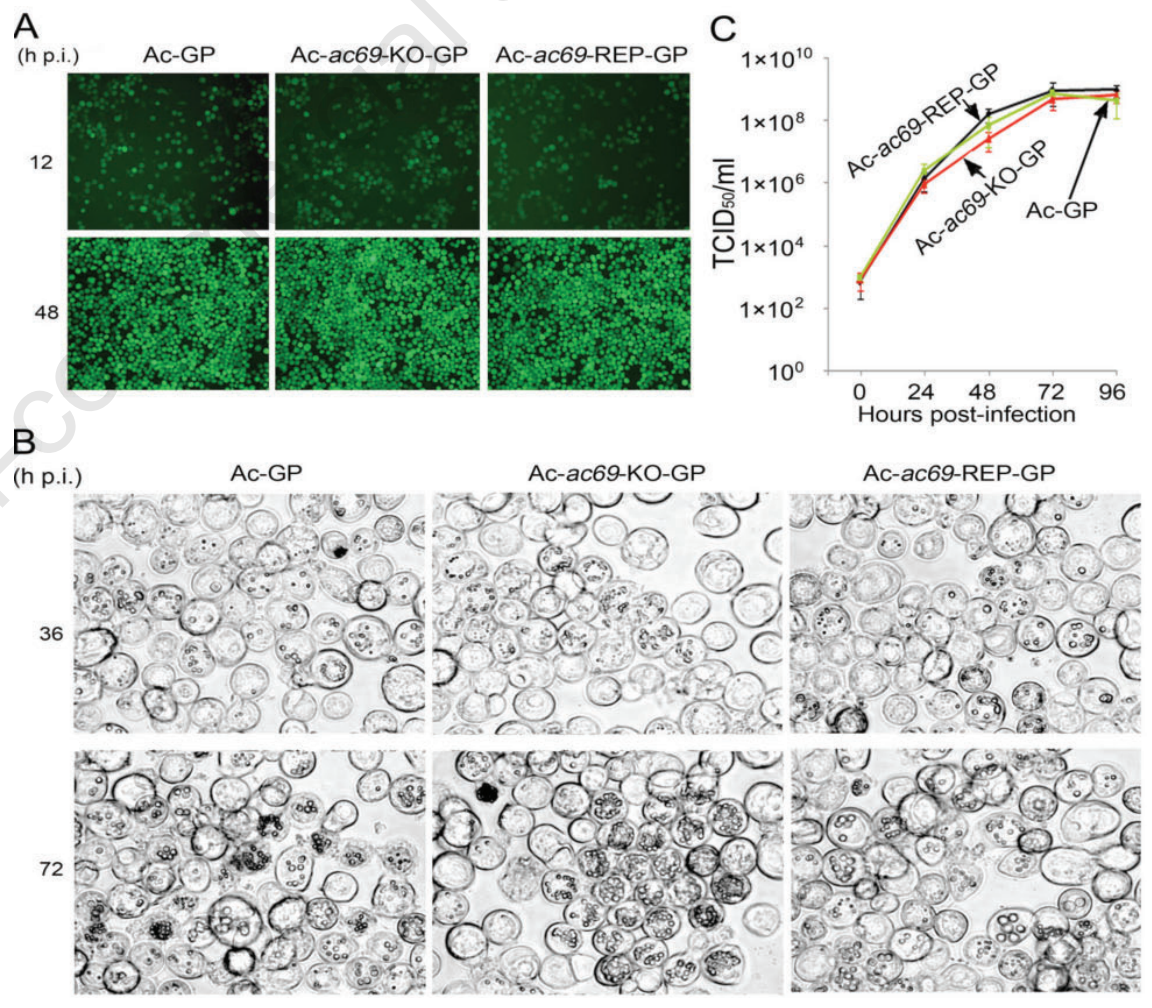

Figure 2. Analysis of viral replication in virus-infected Sf-9 cells. (A) Fluorescence microscopy of Ac-GP, Ac-ac69-KO-GP or Ac-ac69-REP-GP-infected Sf-9 cells at 12 and 48 h p.i. (B) Light microscopy of Ac-GP, Ac-ac69-KO-GP or Ac-ac69-REP-GP-infected Sf-9 cells at 36 and $72 \mathrm{~h}$ p.i. (C) Virus growth curve generated from Ac-GP, Ac-ac69-KOGP or Ac-ac69-REP-GP-infected Sf-9 cells. Sf-9 cells were infected at an MOI of 5 from each virus. Cells culture supernatants were harvested at the selected time points and assayed for the production of infectious virus by $\mathrm{TCID}_{50}$ assay. Each datum point represents the average titer derived from three independent infections. Error bars represent standard errors. 


\section{Discussion}

The AcMNPV ac69 gene, which encodes a protein that contains an S-adenosyl methionine (AdoMet)-dependent methyltransferase signature motif, was first demonstrated to have cap 0-dependent methyltransferase activity by Wu and coworkers, ${ }^{11}$ who postulated that it is one of AcMNPV auxiliary genes, which are not essential for virus replication but nonetheless provide it with some selective advantage, based on the existing ac69 homologs for methylation of viral mRNAs in host and the unaffected budded virus replication level in the ac69-null virus-infected Sf-9 cells. While the hypothesis that ac69 is an auxiliary gene has been partially confirmed in experimental tissue culture systems, it is still not known if ac69 deletion has any effect on ODV synthesis in vitro and virus infectivity in vivo. This study, by comprehensive analyses of an ac69 knockout virus, provides primary evidence that $a c 69$, as a cap 0-dependent methyltransferase, is dispensable for ODV production in Sf-9 cells and virus oral infectivity in T.ni larvae.

In the present study, we describe the use of ET-mediated recombination technology to delete ac69 gene from the AcMNPV genome. This allowed us to investigate the role of AcMNPV ac69 during the AcMNPV virus life cycle. We found that ac69 gene deletion have little effect on the production rates and morphogenesis of budded virus and occlusion body in Sf-9 cells, as evidenced by similar slope of virus growth curve among ac69 wt, knockout, and rescue viruses, and subsequent electron microscopy observation, i.e., the normal rodshaped nucleocapsids associating with electron-dense edges of the virogenic stroma and polyhedra containing 0DVs in ac69 knockout virus-infected cells in comparison with that in wt virus-infected cells. In addition, $\mathrm{LD}_{50}$ and $\mathrm{LT}_{50}$ bioassays performed with T.ni larvae revealed no significant difference between the ac69 knockout virus and wt virus. Together, these results support the above hypothesis that ac69 is an auxiliary gene and suggest that ac 69 may be dispensable for viral virulence or infectivity both in vitro and in vivo.

To date, a number of AcMNPV genes have been shown to be auxiliary genes which can be divided into two categories, genes likely to facilitate virus replication at the cellular level and genes likely to function at the organismal level, ${ }^{9}$ For example the AcMNPV p10 gene, ranged into the former category at the present time, has high expression levels late in the infection process, and has been shown not to be required for viral virulence or infectivity. 9 , 16-18 Although a large number of p10 mutant viruses have now been generated, its role during viral infection is still unclear. AcMNPV genes $v$ fgf and $a c 18$ have been shown not to be essential for viral propagation both in vitro and in vivo. ${ }^{19-21}$ However, lack of $v f g f$ delayed the death time in two host species, Spodoptera frugiperda and T.ni, when the virus was delivered by feeding but not by intrahemocoelic injection, and disruption of ac18 delayed the death time in T.n larvae when the virus was delivered by feeding, suggesting the two genes may play a role in efficient virus infection in vivo. Recently, an ODV-E66 deletion mutant

Table 2. Dose mortality of Ac-GP and Ac-ac69-KO-GP for third-instar T.ni larvae.

\begin{tabular}{lccc} 
Virus & LD $_{50}$ (PIBs per larva) & \multicolumn{2}{c}{$95 \%$ Fiducial limit (PIBs per larva) } \\
& & Lower & Upper \\
Ac-GP & 52.1 & 40.6 & 63.5 \\
Ac-ac69-KO-GP & 46.8 & 23.4 & 68.8 \\
\hline
\end{tabular}

Table 3. Time mortality of Ac-GP and Ac-ac69-KO-GP for third-instar T.ni larvae.

\begin{tabular}{|c|c|c|c|}
\hline \multirow[t]{2}{*}{ Virus } & \multirow[t]{2}{*}{$\mathrm{LT}_{50}(\mathrm{~h})$} & \multicolumn{2}{|c|}{$95 \%$ Fiducial limit (h) } \\
\hline & & Lower & Upper \\
\hline Ac-GP & 83.1 & 81.4 & 84.9 \\
\hline Ac- $a c 69-\mathrm{KO}-\mathrm{GP}$ & 80.1 & 78.3 & 81.9 \\
\hline
\end{tabular}
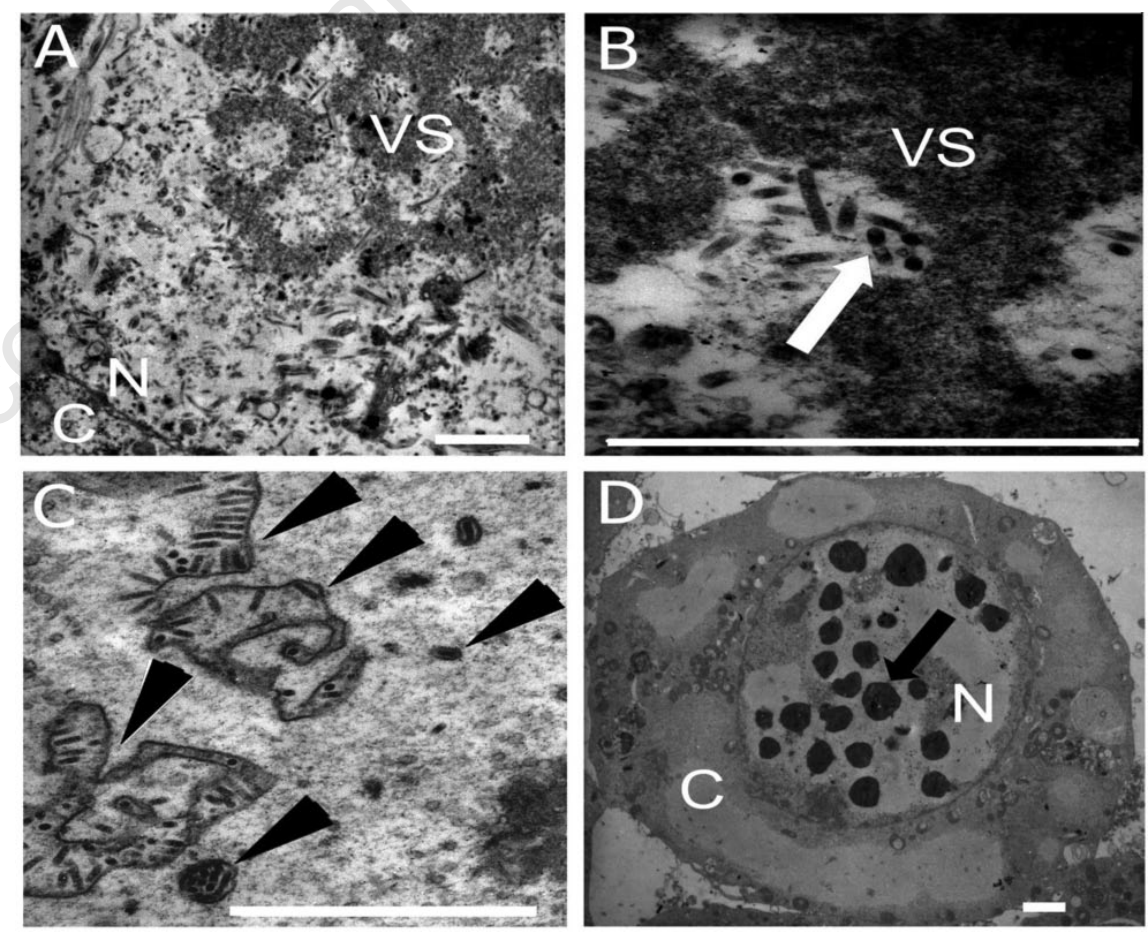

Figure 3. Electron microscopic analysis of Ac-ac69-KO-GP-infected Sf-9 cells to characterize ac69-null mutant. Panels $A$ and $B$ show virogenic stroma enriched with nucleocapsids appearing within the nucleus at $24 \mathrm{~h}$ p.i. Panels $\mathrm{C}$ indicates the aligning of bundles of nucleocapsids with envelopes and their subsequent envelopment to form ODV. Panels $D$ shows occlusion bodies containing ODVs appearing within the nucleus at $72 \mathrm{~h}$ p.i. The nucleus (N), cytoplasm (C), and virogenic stroma (VS) regions are indicated. Triangles indicate bundles of nucleocapsids, ODV in the process of becoming enveloped or fully enveloped ODV. White arrow indicates nucleocapsids in the virogenic stroma. Black arrow indicates occlusion bodies containing ODVs within the nucleus. For A, B, C, D the bar represents $1 \mu \mathrm{m}$. 
As a rule of thumb, viruses will not expend the cost of replication and expressing a gene unless there is some compensating benefit to the virus in terms of increased fitness. Thus it is reasonable to assume that all AcMNPV virus auxiliary genes have some significant role in the viral life cycle. However, the roles of many auxiliary genes, e.g., the AcMNPV p10, ac69 and $P C N A$, are still unclear while they have been tested by a large number of experimental methods. ${ }^{9}$ The reasons why functions for these genes are so difficult to identify may be as follows; first, auxiliary genes are often tested for effects in only one or a small number of host species; second, with current laboratory assay, it is possible that an evolutionally significant increase in fitness might be too small to be reliably detected; and finally, in addition to some generally assayed parameters such as virulence, other parameters that contributes to virus fitness, e.g., ODV stability, host behavior and the ability of the virus to survive as a latent infection, are rarely assayed. Thus, more considerable work, e.g., testing in a larger number of host species and parameters such as ODV stability, is still required to unravel the role of the $a c 69$ gene in AcMNPV life cycle.

Although the exact role of $a c 69$ during the viral infection cycle is still unclear, the present study will lead to a better understanding of the molecular aspects of this protein, and provides a large number of experimental data for the further $a c 69$ gene funtional analysis.

\section{References}

1. Ayres MD, Howard SC, Kuzio J, et al. The complete DNA sequence of Autographa californica nuclear polyhedrosis virus. Virology 1994;202:586-605.

2. Jakubowska AK, Peters SA, Ziemnicka J, et al. Genome sequence of an enhancin gene-rich nucleopolyhedrovirus (NPV) from Agrotis segetum: collinearity with Spodoptera exigua multiple NPV. J Gen Virol 2006;87:537-55.

3. Xiang X, Chen L, Hu X, et al. Autographa californica multiple nucleopolyhedrovirus odv-e66 is an essential gene required for oral infectivity. Virus Res 2011;158:72-8.

4. Blissard GW, Kogan PH, Wei R, Rohrmann GF. A synthetic early promoter from a baculovirus: Roles of the TATA box and conserved start site CAGT sequence in basal levels of transcription. Virology 1992;190:783-93.

5. Pullen SS, Friesen PD. The CAGT motif functions as an initiator element during early transcription of the baculovirus transregulator ie-1. J Virol 1995;69:3575-83.

6. Garcia-Maruniak A, Maruniak JE, Zanotto PMA, et al. Sequence analysis of the genome of the Neodiprion sertifer nucleopolyhedrovirus. J Virol 2004;78:7036-51.

7. Lu A, and Miller LK, in: Miller LK (Ed.). The Baculoviruses. New York: Plenum Press 1997; pp. 193-211.

8. Williams GV, and Faulkner P, in: Miller LK (Ed.). The Baculoviruses. New York: Plenum Press 1997; pp. 61-107.

9. O'Reilly DR, in: Miller LK. (Ed.). The Baculoviruses. New York: Plenum Press 1997; pp. 267-295.

10. Li LL, Harwood SH, Rohrmann GF. Identification of additional genes that influence baculovirus late gene expression. Virology 1999;255:9-19.

11. Wu X, Guarino LA. Autographa californica nucleopolyhedrovirus orf69 encodes an RNA cap (nucleoside-2'-0)-methyltransferase. J Virol, 2003;77:3430-4.

12. Luckow VA, Lee SC, Barry GF, Olins PO. Efficient generation of infectious recombinant baculoviruses by site-specific transposon-mediated insertion of foreign genes into a baculovirus genome propagated in Escherichia coli. J Virol 1993;67:4566-79.

13. Ke J, Wang J, Deng R, Wang X. Autographa californica multiple nucleopolyhedrovirus ac66 is required for the efficient egress of nucleocapsids from the nucleus, general synthesis of preoccluded virions and occlusion body formation. Virology 2008;374: 421-31.

14. Li Y, Wang JW, Deng RQ, et al. vlf-1 Deletion Brought AcMNPV to Defect in Nucleocapsid Formation. Virus Genes 2005; 31:275-84.

15. 0'Reilly DR, Miller LK, Luckow VA. Baculovirus expression vector: a laboratory manual. New York: WH Freeman and Co.; 1992.

16. Adang MJ, Miller LK. Molecular cloning of DNA complementary to mRNA of the baculovirus Autographa californica nuclear polyhedrosis virus: Location and gene products of RNA transcripts found late in infection. J Virol 1982;44:782-93.

17. Smith GE, Vlak JM, Summers MD. In Vitro Translation of Autographa californica Nuclear Polyhedrosis Virus Early and Late mRNAs. J Virol 1982;44:199-208.

18. Vlak JM, Klinkenberg FA, Zaal KJM, et al. Functional studies on the p10 gene of Autographa californica nuclear polyhedrosis virus using a recombinant expressing a p10-beta-galactosidase fusion gene. Gen Virol 1988;69:765-76.

19. Detvisitsakun C, Hutfless EL, Berretta MF, Passarelli AL. Analysis of a baculovirus lacking a functional viral fibroblast growth factor homolog. Virology 2006;346:258-65.

20. Detvisitsakun C, Cain EL, Passarelli AL. The Autographa californica M nucleopolyhedrovirus fibroblast growth factor accelerates host mortality. Virology 2007;365: 70-8.

21. Wang Y, Wu W, Li Z, et al. ac18 is not essential for the propagation of Autographa californica multiple nucleopolyhedrovirus. Virology 2007;367:71-81. 